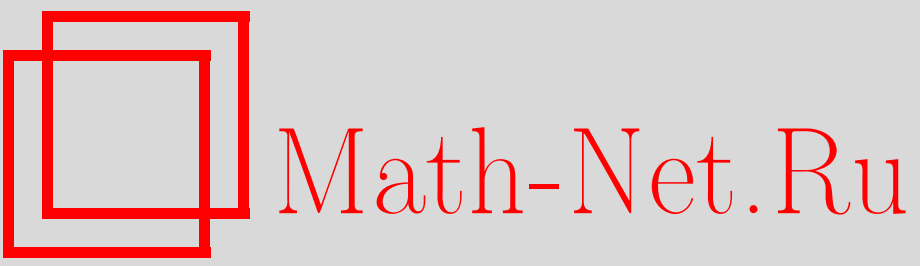

О. С. Якимова, О слабо коммутативных однородных пространствах, УМН, 2002, том 57, выпуск 3, 171-172

DOI: https://doi.org/10.4213/rm527

Использование Общероссийского математического портала Math-Net.Ru подразумевает, что вы прочитали и согласны с пользовательским соглашением

http://www.mathnet.ru/rus/agreement

Параметры загрузки:

IP : 34.229 .45 .116

26 апреля 2023 г., $17: 18: 20$ 


\title{
О СЛАБО КОММУТАТИВНЫХ ОДНОРОДНЫХ ПРОСТРАНСТВАХ
}

\author{
О. С. ЯкимовА
}

Пусть $X=G / K-$ связное риманово однородное пространство группы Ли $G$. Будем предполагать, что действие $G: X$ локально эфффективно. Пусть $\mathfrak{g}=\operatorname{Lie} G, \mathfrak{k}=\operatorname{Lie} K$.

Обозначим через $\mathscr{P}\left(T^{*} X\right)^{G}$ алгебру $G$-инвариантных функций на кокасательном расслоении $T^{*} X$, полиномиальных на слоях; через $\mathscr{D}(X)^{G}$ - алгебру $G$-инвариантных диффференциальных операторов на $X$. Как известно (см. [1]), $\mathscr{P}\left(T^{*} X\right)^{G}=\operatorname{gr} \mathscr{D}(X)^{G} \cong S(\mathfrak{g} / \mathfrak{k})^{K}$. На алгебре $\mathscr{P}\left(T^{*} X\right)^{G}$ естественньм образом определена скобка Пуассона. Можно показать, что она с точностью до знака совпадает со скобкой на $S(\mathfrak{g} / \mathfrak{k})^{K} \cong S(\mathfrak{g})^{K} /(S(\mathfrak{g}) \mathfrak{k})^{K}$. Здесь $(S(\mathfrak{g}) \mathfrak{k})^{K}$-пуассонов идеал алгебры $S(\mathfrak{g})^{K}$.

ОПРедЕлЕниЕ 1. Однородное пространство $X$ назьвается слабо коммутативным, если алгебра $\mathscr{P}\left(T^{*} X\right)^{G}$ (или $\left.S(\mathfrak{g} / \mathfrak{k})^{K}\right)$ коммутативна относительно скобки Пуассона.

Слабо коммутативные однородные пространства редуктивных групп являются вещественными формами однородных афффинных сферических многообразий, см. [1]. Они классифицированы с точностью до локального изоморфизма в работе [2].

Приведем здесь некоторые свойства слабо коммутативных пространств, доказанные в [1].

Пусть $\mathfrak{k}^{0}=(\mathfrak{g} / \mathfrak{k})^{*}, \mathfrak{n}=\operatorname{Lie} N, \mathfrak{l}=\operatorname{Lie} L$. Однородное пространство $X=G / K$ слабо коммутативно тогда и только тогда, когда для точки общего положения $\alpha \in \mathfrak{k}^{0}$ выполнено условие:

(1) $\operatorname{ad}^{*}(\mathfrak{g}) \alpha \cap \mathfrak{k}^{0}=\operatorname{ad}^{*}(\mathfrak{k}) \alpha$.

Пусть $X=G / K$ - односвязное слабо коммутативное пространство связной группы Ли $G$. Тогда $G=N \lambda L$, где $N$ - не более чем 2-ступенно нильпотентная односвязная группа Ли, $L-$ связная редуктивная подгруппа группы $G$, содержащая $K$, и выполнено условие

(i) $\mathbb{R}[\mathfrak{n}]^{L}=\mathbb{R}[\mathfrak{n}]^{K}$.

Слабо коммутативные пространства, в которых $L=K$, назьваются пространствами гейзенбергова типа. В [1] получена их классификация при условии, что представление $K: \mathfrak{n} / \mathfrak{n}^{\prime}$ неприводимо.

Лемма. Пусть пространство $G / K$ слабо коммутативно $и[\mathfrak{n}, \mathfrak{n}] \neq 0$. Рассмотрим коммутативную односвязную группу $\widehat{N}$, касательная алгебра которой изоморфна $\mathfrak{n}$ как L-модуль. Тогда однородное пространство $\widehat{G} / K$ группь $\widehat{G}=\widehat{N} 入 L$ тоже слабо коммутативно.

ДокАЗАТЕльство. На $S(\mathfrak{g} / \mathfrak{k})$ определена $K$-инвариантная биградуировка $S^{n, l}(\mathfrak{g} / \mathfrak{k})=$ $S^{n}(\mathfrak{n}) S^{l}(\mathfrak{l} / \mathfrak{k})$. Заметим, что если $a \in S^{n, l}, b \in S^{n^{\prime}, l^{\prime}}\left(a, b \in S(\mathfrak{g} / \mathfrak{k})^{K}\right)$, то

$$
\{a, b\}=\{a, b\}_{\mathfrak{l}}+\{a, b\}_{\mathfrak{n}}, \quad \text { где }\{a, b\}_{\mathfrak{l}} \in S^{n+n^{\prime}, l+l^{\prime}-1}, \quad\{a, b\}_{\mathfrak{n}} \in S^{n+n^{\prime}-1, l+l^{\prime}} .
$$

Так как $K$-модули $\mathfrak{g}$ и $\widehat{\mathfrak{g}}$ изоморфны, имеем $S(\mathfrak{g} / \mathfrak{k})^{K}=S(\widehat{\mathfrak{g}} / \mathfrak{k})^{K}$. Пусть $\widehat{a}, \widehat{b}-$ биоднородные элементы $a, b$ пространства $S(\mathfrak{g} / \mathfrak{k})^{K}$, рассматриваемые как элементы алгебры $S(\widehat{\mathfrak{g}} / \mathfrak{k})^{K}$. Тогда $\{\widehat{a}, \widehat{b}\}=\{a, b\}_{\mathfrak{l}}=0$.

Пусть $\mathfrak{l}=\mathfrak{k} \oplus \mathfrak{m}-K$-инвариантное разложение. Тогда $\mathfrak{k}^{0}=\mathfrak{m}^{*} \oplus \mathfrak{n}^{*}$.

Из условия (i) следует, что орбиты групп $L$ и $K$ в $\mathfrak{n}$ совпадают, $L$-модули $\mathfrak{n}$ и $\mathfrak{n}^{*}$ изоморфны,

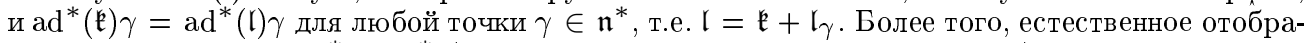
жение ограничения $\tau: \mathfrak{l}^{*} \rightarrow \mathfrak{l}_{\gamma}^{*}$ (являющееся гомоморфизмом $L_{\gamma}$-модулей) задает изоморфизм

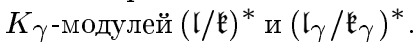

Работа выполнена при частичной поддержке C.R.D.F. (грант RM1-2088). 
Tеорема 1. Однородное пространство $X=G / K$ слабо коммутативно тогда и только тогда, когда выполнено условие (i), а также следующие условия:

(ii) для точки общего положения $\gamma \in \mathfrak{n}^{*}$ пространство $L_{\gamma} / K_{\gamma}$ слабо коммутативно;

(iii) для точки общего положения $\beta \in \mathfrak{m}^{*}$ пространство $\left(K_{\beta} \curlywedge N\right) / K_{\beta}$ слабо коммутативно.

ДокАЗАтельство. Как уже отмечалось, условие (i) является необходимым. Будем предполагать, что оно выполнено.

$(\Longleftarrow)$ Покажем, как доказьвается достаточность указанных условий в случае $[\mathfrak{n}, \mathfrak{n}]=0$.

Пусть $\alpha=\beta+\gamma \in \mathfrak{k}^{0}, \beta \in \mathfrak{m}^{*}, \gamma \in \mathfrak{n}^{*}$. Достаточно доказать, что если условие (1) выполнено для тройки $\left(L_{\gamma}, K_{\gamma}, \beta\right)$, то оно выполнено и для $(G, K, \alpha)$.

Пусть $l \in \mathfrak{l}, n \in \mathfrak{n}$ и $\operatorname{ad}^{*}(l+n) \alpha \in \mathfrak{k}^{0}$. Найдем такой элемент $k \in \mathfrak{k}$, что $\operatorname{ad}^{*}(l+n-k) \alpha=0$. Так как $\mathfrak{l}=\mathfrak{l}_{\gamma}+\mathfrak{k}$, можно считать, что $l \in \mathfrak{l}_{\gamma}$. Посколшку, кроме того, $\operatorname{ad}^{*}(n) \beta=0$, имеем $\operatorname{ad}^{*}(l+n) \alpha=\operatorname{ad}^{*}(l) \beta+\operatorname{ad}^{*}(n) \gamma$. Заметим, что $\operatorname{ad}^{*}(n) \gamma\left(\mathfrak{k}_{\gamma}\right) \in \operatorname{ad}^{*}(\mathfrak{k})_{\gamma}(n)=0$. Следовательно, $\operatorname{ad}^{*}(l) \beta\left(\mathfrak{k}_{\gamma}\right)=0$. Для тройки $\left(L_{\gamma}, K_{\gamma}, \beta\right)$ выполнено условие (1), т.е.

$$
\exists k \in \mathfrak{k}_{\gamma}: \operatorname{ad}^{*}(l-k) \beta\left(\mathfrak{l}_{\gamma}\right)=0 .
$$

Непосредственным вычислением можно убедиться в том, что элемент $k$ действительно удовлетворяет нашему условию.

Вернемся к конструкции, рассмотренной в лемме. Теперь известно, что пространство $\widehat{G} / K$ слабо коммутативно. Докажем, что тогда из условия (iii) следует слабая коммутативность пространства $G / K$. Достаточно доказать, что $\{a, b\}_{\mathfrak{n}}=0$ для любых двух биоднородных элементов $a, b \in S(\mathfrak{g} / \mathfrak{k})^{K}$. Пусть $\beta \in \mathfrak{m}^{*}$. Определим отображение

$$
\varphi_{\beta}: S(\mathfrak{g} / \mathfrak{k}) \rightarrow S(\mathfrak{g} / \mathfrak{k}) /(\xi-\beta(\xi): \xi \in \mathfrak{m}) \cong S(\mathfrak{n}) .
$$

Легко видеть, что $\varphi_{\beta}\left(S(\mathfrak{g} / \mathfrak{k})^{K}\right) \subset S(\mathfrak{n})^{K}$. Заметим, что для точки общего положения $\beta \in \mathfrak{m}^{*}$ $\varphi_{\beta}\left(\{a, b\}_{\mathfrak{n}}\right)=\left\{\varphi_{\beta}(a), \varphi_{\beta}(b)\right\}=0$. Следовательно, и $\{a, b\}_{\mathfrak{n}}=0$.

$(\Longrightarrow)$ (ii) Достаточно доказать, что если $\beta \in \mathfrak{m}^{*}, \gamma \in \mathfrak{n}^{*}$ и для точки $\alpha=\beta+\gamma \in \mathfrak{k}^{0}$ выполнено условие (1), то условие (1) вьполнено для тройки $\left(L_{\gamma}, K_{\gamma}, \beta\right)$.

Можно считать алгебру $\mathfrak{n}$ коммутативной. Пусть $\bar{\beta} \in\left(\mathfrak{l}_{\gamma} / \mathfrak{k}_{\gamma}\right)^{*}, l \in \mathfrak{l}_{\gamma}$ и $\operatorname{ad}^{*}(l) \bar{\beta}\left(\mathfrak{k}_{\gamma}\right)=0$. Нужно найти такой элемент $k \in \mathfrak{k}_{\gamma}$, для которого $\operatorname{ad}^{*}(l) \bar{\beta}=\operatorname{ad}^{*}(k) \bar{\beta}$. В пространстве $\mathfrak{m}$ существует единственный элемент $\beta$, для которого $\tau(\beta)=\bar{\beta}$. Заметим, что $\left.\operatorname{ad}^{*}(l) \beta\right|_{\mathfrak{k}} \in\left(\mathfrak{k} / \mathfrak{k}_{\gamma}\right)^{*}=$ $\left.\operatorname{ad}^{*}(\mathfrak{n}) \gamma\right|_{\mathfrak{k}}$. Поэтому найдется такой элемент $n \in \mathfrak{n}$, что $\operatorname{ad}^{*}(l+n)(\beta+\gamma)=\operatorname{ad}^{*}(l) \beta+\operatorname{ad}^{*}(n) \gamma \in \mathfrak{k}^{0}$. Тогда существует элемент $k \in \mathfrak{k}$, для которого $\mathrm{ad}^{*}(l+n-k)(\beta+\gamma)=0$. При ограничении на $\mathfrak{l}_{\gamma}$ имеем: $\left.\operatorname{ad}^{*}(l+n)(\beta+\gamma)\right|_{\mathfrak{l}_{\gamma}}=\left.\operatorname{ad}^{*}(l) \beta\right|_{\mathfrak{l}_{\gamma}}=\left.\operatorname{ad}^{*}(k) \beta\right|_{\mathfrak{l}_{\gamma}}$. Осталось убедиться в том, что $\operatorname{ad}^{*}(k) \gamma=0$. Действительно, $[\mathfrak{n}, \mathfrak{n}]=0 \Longrightarrow\left(\operatorname{ad}^{*}(l) \beta+\operatorname{ad}^{*}(n) \gamma\right)(\mathfrak{n})=0=\operatorname{ad}^{*}(k) \gamma(\mathfrak{n}) \Longrightarrow$ $\operatorname{ad}^{*}(k) \gamma=0$.

(iii) Пусть $\beta \in \mathfrak{m}^{*}$. Покажем, что алгебра $S(\mathfrak{n})^{K}$ коммутативна относительно скобки Пуассона. Достаточно доказать, что отображение $\varphi_{\beta}$ сюръективно. Как известно, $S(\mathfrak{g})=\mathbb{R}\left[\mathfrak{g}^{*}\right]$, $S(\mathfrak{g} / \mathfrak{k})=\mathbb{R}\left[\mathfrak{k}^{0}\right]$. Заметим, что $\mathfrak{n}^{*} / / K_{\beta} \cong\left(K \beta \oplus \mathfrak{n}^{*}\right) / / K \subset \mathfrak{k}^{0} / / K$. Так как орбита $K \beta$ замкнута в $\mathfrak{m}^{*}$, подмножество $\left(K \beta \oplus \mathfrak{n}^{*}\right) / / K$ замкнуто в $\mathfrak{k}^{0} / / K$. Поэтому отображение ограничения $\mathbb{R}\left[\mathfrak{k}^{0}\right]^{K} \rightarrow \mathbb{R}\left[K \beta \oplus \mathfrak{n}^{*}\right]^{K}$, а следовательно, и $\varphi_{\beta}$ сюръективно.

Отметим, что условие (i) равносильно равенству $\mathfrak{l}=\mathfrak{l}_{\gamma}+\mathfrak{k}$, для точки общего положения $\gamma \in \mathfrak{n}^{*}$, а группа $L_{\gamma}$ - редуктивна. Таким образом, проверка слабой коммутативности сводится к проверке условия (iii). По-видимому, с помощью теоремы 1 можно получить полную классификацию слабо коммутативных пространств.

\section{СПИСОК ЛИТЕРАТУРЫ}

[1] Э. Б. Винберг // УМН. 2001. Т. 56. №1. С. 3-62. [2] О. С. Якимова // Вестн. МГУ. Сер. Матем., мех. 2002. № 2 (в печати). 